\title{
Richard Hooker, l'hérésie papiste et un protestantisme anglais de la continuité
}

Richard Hooker, Papist Heresy and the Catholic Continuity of English

Protestantism

Rémy Bethmont

\section{CpenEdition}

Journals

Édition électronique

URL : http://journals.openedition.org/rfcb/3567

DOI : $10.4000 /$ rfcb.3567

ISSN : 2429-4373

Éditeur

CRECIB - Centre de recherche et d'études en civilisation britannique

Édition imprimée

Date de publication : 1 mars 2013

ISBN : 2-911580-37-0

ISSN : 0248-9015

Référence électronique

Rémy Bethmont, " Richard Hooker, l'hérésie papiste et un protestantisme anglais de la continuité », Revue Française de Civilisation Britannique [En ligne], XVIII-1 | 2013, mis en ligne le 01 mars 2013, consulté le 21 mars 2020. URL : http://journals.openedition.org/rfcb/3567 ; DOI : https://doi.org/ $10.4000 /$ rfcb.3567

Ce document a été généré automatiquement le 21 mars 2020.

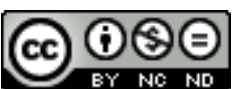

Revue française de civilisation britannique est mis à disposition selon les termes de la licence Creative Commons Attribution - Pas d'Utilisation Commerciale - Pas de Modification 4.0 International. 


\title{
Richard Hooker, l'hérésie papiste et un protestantisme anglais de la continuité
}

\author{
Richard Hooker, Papist Heresy and the Catholic Continuity of English \\ Protestantism
}

Rémy Bethmont

1 La vie de Richard Hooker (1554-1600) que publie Isaac Walton en 1662 fait de ce théologien élisabéthain, assez peu lu de son vivant, le grand défenseur de l'orthodoxie anglicane, c'est-à-dire d'une Réforme modérée entre les "erreurs» de Rome et les « excès » genevois. Hooker devient dès lors le champion de la via media anglicane et son magnum opus, Of the Laws of Ecclesiastical Polity ${ }^{1}$, est depuis considéré par les anglicans comme une somme théologique de référence pour comprendre et définir leur conception de l'Église.

2 Les recherches récentes ont néanmoins montré que cette vision de Hooker comme défenseur d'une orthodoxie anglicane à mi-chemin entre Rome et Genève est bien plus le produit de la réécriture de l'histoire effectuée à la Restauration par les épiscopaliens triomphants que le reflet de ce que représente la position de Hooker dans le contexte $\mathrm{du} \mathrm{XVI} \mathrm{I}^{\mathrm{e}}$ siècle finissant ${ }^{2}$. Loin d'être le porte-parole autorisé et reconnu de l'orthodoxie nationale élisabéthaine, il frappe surtout ses contemporains par son originalité. Sa voix n'est qu'une composante parmi d'autres dans une conversation théologique et ecclésiologique à laquelle contribuent divers courants puritains et conformistes dont les lignes de séparation sont mouvantes et complexes. Le conformisme de Hooker n'est ainsi pas celui de l'archevêque Whitgift qui, sur la prédestination ou sur la question de l'autorité des Écritures, est plus proche des puritains ${ }^{3}$. Mais pour les anglicans du dernier tiers du XVII ${ }^{e}$ siècle et leur postérité, Hooker constitue le maillon qui les rattache aux origines de la Réforme anglaise; on se soucie peu du fait que ce maillon n'ait pas été, à la fin du XVI e siècle, la seule manière (ni même la manière majoritaire) de concevoir et de défendre le dispositif ecclésial élisabéthain. L'importance de Hooker pour l'anglicanisme (concept pourtant inconnu à son époque !) doit se chercher dans la 
manière qu'il a de défendre un mode d'être protestant, qui refuse la radicalisation de l'opposition entre les deux grands blocs religieux de l'Europe, "catholique » et " protestant », dans les décennies qui suivent le Concile de Trente.

3 Les formes de la religion élisabéthaine reflètent un état de la Réforme qui est celui des années 1540 , mais qui, dans les années 1580 , est déjà fort dépassé et en porte-à-faux avec les avancées de la Réforme protestante sur le continent à la fin du XVI ${ }^{e}$ siècle ${ }^{4}$. Hooker ressent le besoin, pour défendre ce qui, pour les puritains, apparait comme un anachronisme incompréhensible, de recourir à une argumentation novatrice qui puisse parler à sa génération. Autant dans sa méthode ${ }^{5}$ que dans le contenu de son discours, il vante les avantages d'une Réforme qui a gardé tout ce qu'elle pouvait de l'Église médiévale, inaugurant un discours protestant de la continuité ${ }^{\text {contre un }}$ protestantisme de la rupture. Il s'appuie sur une compréhension assez originale, dans le contexte réformé, de l'hérésie papiste, à laquelle il n'attribue qu'une capacité limitée à détruire la continuité historique de la véritable Église catholique et apostolique. Ce faisant, il théorise un protestantisme à l'imagination ecclésiale sensiblement différente de celle qui se développe dans les Églises Réformées de Suisse et de France et à laquelle les puritains souscrivent avec enthousiasme. C'est cet autre protestantisme hookérien dans lequel, plus tard, les anglicans puiseront pour définir une via media qui donne à l'anglicanisme un caractère œcuménique, inclassable dans le paysage religieux occidental depuis le XVI siècle.

\section{L'hérésie de Rome et le salut des papistes}

4 La définition que donne Hooker de l'hérésie romaine est centrale dans la construction, chez lui, d'un discours protestant de la continuité. La controverse qui accompagne sa production et oppose Hooker au puritain Walter Travers en 1586 met en relief le caractère marginal, décalé de ce discours dans le protestantisme anglais de l'époque. Dans les dernières décennies du XVI ${ }^{\mathrm{e}}$ siècle, la vigueur des attaques romaines contre l'Église d'Angleterre se fait sentir, à un moment où la réforme tridentine a donné de nouvelles forces à la cause de Rome. Un des arguments de la propagande papiste contre la Réforme est que celle-ci condamne à la damnation toutes les générations antérieures, faisant ainsi de la doctrine réformée une religion contraire à tout sentiment de piété filiale. Maints théologiens protestants se sentent poussés à contre-attaquer, et Hooker se joint à l'entreprise dans une série de sermons sur le livre d'Habakuk qui visent à réfuter les critiques papistes. Quelle que soit l'originalité argumentative de ces sermons, leur conclusion reflète le consensus réformé : tous les ancêtres papistes des protestants anglais n'ont pas été empêchés de trouver le salut, malgré l'erreur dans laquelle l'Église médiévale hérétique les induisait?

5 Au départ, dans le premier sermon, rien ne laisse supposer que Hooker s'écarte du discours réformé ambiant. Mais alors qu'il est attaqué par Travers sur ce qui est probablement un écart involontaire de langage ${ }^{8}$, il est conduit à préciser sa pensée et à examiner plus avant la question du salut des papistes dans les deux sermons de la série qui nous sont restés dans leur intégralitée. C'est là qu'apparaît l'originalité de Hooker. Au lieu d'en rester à invoquer le mystère de la grâce divine qui a pu, d'une manière ou d'une autre, sauver certains membres des générations papistes antérieures à la Réforme, comme le fait Oliver Carter ${ }^{10}$, Hooker cherche à expliquer comment il est théologiquement possible que Dieu ait pu sauver des hérétiques ${ }^{11}$. Plutôt que de 
suspendre son jugement sur les papistes du passé, Hooker cherche à démontrer la possibilité de leur salut ${ }^{12}$. Cette originalité argumentative, nous le verrons, est à l'origine d'une conception de l'Église en décalage avec l'opinion réformée majoritaire, dans laquelle se reconnaissent les anglicans de la Restauration et leur postérité.

L'argument de Hooker relatif au salut des papistes repose tout en entier sur la distinction entre rejet direct et indirect du fondement de la foi qu'est le salut par le Christ seul ${ }^{13}$. Les papistes affirment ce fondement même si, dit Hooker, ils le pervertissent en prêchant la nécessité des œuvres pour que le salut soit appliqué à l'individu, obscurcissant ainsi chez lui le fait que c'est la seule œuvre du Christ qui sauve. Le résultat est que les papistes, par effet de conséquence (et non directement), renversent le fondement de la foi ('they overthrow the very foundation of faith by consequent ${ }^{14}$ ). Hooker cherche alors à démontrer que l'Église médiévale, malgré ses corruptions, avait gardé le fondement de la foi de manière suffisante pour qu'il ne soit pas impossible de trouver le salut.

7 Hooker revient sur le concept d'hérésie, qu'il divise en plusieurs catégories. La première est une négation explicite et consciente d'un article du Credo :

We make a difference of heresies; accounting them in the next degree to infidelity, which directly deny any one thing to be which is expressly acknowledged in the articles of our belief [...] As for example; if a man should say, 'There is no catholic Church,' it followeth immediately hereupon, that this Jesus, whom we call the Saviour, is not the Saviour of the world, because [...] the true Messias should [...] gather such a Church as is catholic, not restrained any longer unto one circumcised nation.

8 La deuxième est une négation plus indirecte mais vraisemblablement tout aussi consciente des articles de foi :

In a second rank we place them, out of whose positions the denial of any of the foresaid articles may be with like facility concluded; such are they which have denied, either the divinity of Christ, with Hebion, or with Marcion, his humanity.

9 La troisième, dans laquelle Hooker place l'hérésie papiste, est une négation plus indirecte encore et, dans bien des cas au Moyen Âge, inconsciente :

Heresies there are of a third sort, such as the church of Rome maintaineth, which being removed by a greater distance from the foundation, although indeed they overthrow it; yet because of that weakness, which the philosopher noteth in men's capacities when he saith, that the common sort cannot see things which follow in reason, when they follow, as it were afar off by many deductions; therefore the repugnancy between such heresy and the foundation is not so quickly nor so easily found, but that an heretic of this, sooner than of the former kind, may directly grant, and consequently nevertheless deny, the foundation of faith. ${ }^{15}$

Bien que l'erreur de Rome ne soit pas mise dans la même catégorie (la deuxième) que les grandes hérésies combattues par les Pères de l'Église, cela ne veut pas dire qu'elle est moins grave, puisqu'elle renverse, tout autant que la deuxième, le fondement de la foi. Hooker ne cesse d'ailleurs d'affirmer tout au long de son sermon que la doctrine romaine conduit à la damnation. Mais son propos est de montrer que l'erreur de Rome est configurée de telle manière qu'elle n'apparaît pas aussi facilement que les autres comme hérétique, en particulier aux yeux du commun dont la puissance de réflexion ne lui permet pas de déduire par un long raisonnement qu'il y a négation des articles du Credo. Si bien que le papiste du peuple, peu versé dans les subtilités théologiques, peut très bien croire affirmer le fondement de la foi en adhérant à une doctrine qui pourtant 
le renverse. C'est cette ignorance innocente de la pure doctrine que Dieu pardonne pour les générations passées, nées trop tôt pour bénéficier des lumières de la Réforme.

\section{L'Église papiste médiévale, passeur de la foi apostolique}

11 Si Hooker, dans aucun de ses écrits, ne va jamais jusqu'à étendre à la Rome de son temps la possibilité du salut, un pas a bien été franchi, qui informe sa compréhension de l'Église dans of the Laws of Ecclesiastical Polity. Comme le montre fort bien Deborah Shuger, Hooker, dans son sermon sur la justification, a étendu le champ d'application de la doctrine réformée de la persévérance des élus. À l'idée que les péchés de ces derniers ne les coupent jamais définitivement de la grâce, on ajoute celle de l'impossibilité pour l'erreur doctrinale de constituer un obstacle définitif au salut. Quels que soient non seulement les péchés mais aussi les erreurs de ses élus, Dieu les ramènera toujours, en fin de compte, au salut. L'hérésie, en d'autres termes, n'est pas nécessairement signe d'une absence d'élection de la part de Dieu, à partir du moment où il n'y a pas reniement intérieur du Christ de la part de l'individu. C'est dire que la notion de foi justifiante chez Hooker est "radicalement minimaliste ${ }^{16}$, puisque l'hérésie du troisième type caractérisant le papisme, hérésie objective mais sans intention de se séparer du fondement de la foi, n'est pas en soi un obstacle à la justification. La Rome médiévale était donc bien toujours l'Église du Christ, malgré son hérésie.

12 La différence qui sépare Hooker de la tradition argumentative calviniste est nette. Pour celle-ci, en effet, si Rome peut encore être qualifiée d'Église, c'est pour une raison toute négative :

Daniel et sainct Paul ont prédit que l'Antéchrist seroit assis au temple de Dieu (Dan. 9:27 ; 2 Thess. 2:4) : nous disons que le Pape est le capitaine de ce règne maudit et exécrable, pour le moins en l'Eglise occidentale. Puisqu'il est dit que le siège de l'Antéchrist sera au temple de Dieu, par cela il est signifié que son règne sera tel, qu'il n'abolira point le nom de Christ ne de son Eglise. De là il appert que nous ne nions point que les Eglises sur lesquelles il domine par sa tyrannie, ne demeurent Eglise. ${ }^{17}$

13 Le discours de Hooker, qui qualifie lui aussi le pape d'antéchrist, n'est pas absolument incompatible avec celui de Calvin, mais le théologien anglais a une manière bien différente de discerner en Rome l'Église du Christ. En distinguant l'hérésie objective du papisme de l'intention innocente qui a pu la soutenir, Hooker donne moins d'importance à la notion de pureté doctrinale dans la définition de la vraie Église que dans la pensée réformée classique.

On en voit la conséquence dans le livre IV des Lois. Hooker défend les cérémonies dont l'Église d'Angleterre perpétue l'usage, en commun avec Rome, contre les puritains qui veulent au contraire purger l'Église de tout ce qui peut rappeler le papisme hérétique. Les puritains ne conçoivent la nécessaire réforme de l'Église que comme une rupture. Leur argument se résume ainsi : 'Evils must be cured by their contraries; and therefore the cure of the Church infected with the poison of Antichristianity must be done by that which is thereunto as contrary as may be ${ }^{18}$. Hooker, au contraire, tient un discours où Réforme et continuité avec un passé corrompu ne sont pas entièrement antithétiques. 

l'habitude de le concevoir, que l'Église romaine médiévale a été un passeur de la foi et de la pratique apostoliques. Il déclare : 'We have received from them nothing, but that which they did themselves receive from such, as we cannot deny to have been the people of God, yea such, as either we must acknowledge for our own forefathers or else disdain the race of Christ' ${ }^{19}$ et, un peu plus loin :

The ceremonies which we have taken from such as were before us, are not things that belong to this or that sect, but they are the ancient rites and customs of the Church of Christ, whereof ourselves being a part, we have the selfsame interest in them which our fathers before us had, from whom the same are descended unto us. 20

16 Ainsi, le discours de retour à la pureté de l'Église primitive, habituel dans tout le protestantisme, se superpose, partiellement certes mais avec une réelle insistance, à celui de l'héritage dans la continuité. À l'insistance puritaine sur l'unité des Églises réformées (qui se traduit par la demande que l'église d'Angleterre cesse de s'isoler en refusant d'imiter les formes de culte genevoises) ${ }^{21}$, Hooker ajoute une unité historique avec tout ce que le passé de l'Église a transmis de la doctrine et de la pratique apostoliques, y compris dans les périodes les plus corrompues.

\section{Un protestantisme dans la continuité historique catholique}

L'orthodoxie nationale que défend Hooker se situe donc dans une continuité maximale avec le passé médiéval en tout ce qui participe des adiaphora (les choses indifférentes au salut); on n'a réformé que ce qui renversait le fondement de la foi par conséquence, à savoir tout ce qui obscurcissait, dans la doctrine et la pratique chrétiennes, la justification par la foi en Christ sans les œuvres. La continuité avec le passé est d'autant plus importante qu'elle est porteuse d'une autorité pour ainsi dire "naturelle » qui peut guider le commun dans la droiture et la piété. Trop de réforme peut finir par tuer tout véritable esprit de religion dans le peuple ${ }^{22}$ :

The Church of England being to alter her received laws concerning such orders, rites, and ceremonies, as had been in former times an hindrance unto piety and religious service of God, was to enter into consideration first, that the change of laws, especially concerning matter of religion, must be warily proceeded in [...] The wisdom which is learned by tract of time, findeth the laws that have been in former ages established, needful in later to be abrogated [...] But true withal it is, that alteration though it be from worse to better hath in it inconveniences, and those weighty $[. .$.$] if it be a law which the custom and continual practice of many ages or$ years hath confirmed in the minds of men, to alter it must needs be troublesome and scandalous. It amazeth them, it causeth them to stand in doubt whether any thing be in itself by nature either good or evil [...] when they behold even those things disproved, disannulled, rejected, which use had made in a manner natural. What have we to induce men unto the willing obedience and observation of laws, but [...] the weight of that long experience, which the world hath had thereof with consent and good liking? So that to change any such law must needs with the common sort impair and weaken the force of those grounds, whereby all laws are made effectual. ${ }^{23}$

18 Au-delà du conservatisme politique évident de Hooker, pour qui les réformes religieuses répétées pourraient encourager la désobéissance, voire la rébellion, contre

Revue Française de Civilisation Britannique, XVIII-1 | 2013 
l'autorité de la Couronne, qui assure la stabilité à la fois politique et religieuse du royaume ${ }^{24}$, Hooker démontre sa préoccupation pour l'Église visible en tant que société, et plus particulièrement ici, que société anglaise ${ }^{25}$. La nécessaire recherche de la pureté doctrinale doit parfois conduire à réformer les rites et les pratiques de l'Église, mais les réformes (même nécessaires) constituent toujours un certain danger pour la société ecclésiale. Celle-ci, en effet, à l'instar des papistes des générations passées, apprend la vie de foi authentique autant par l'enseignement prodigué par les pasteurs que, de façon plus osmotique, en se laissant modeler par les pratiques cultuelles transmises d'une génération à l'autre.

Rowan Williams, dans un article sur la théologie de Hooker, attire l'attention sur le fait que la liturgie a chez lui une valeur propre, distincte de celle de l'instruction rationnelle, telle qu'un sermon, par exemple, peut la dispenser : 'Public worship deals with the whole of that humanity assumed by Christ, its frailty and physicality. We are to be concerned about something more than ideas in worship because the act of God in Christ is more than the conveying of information to $\mathrm{us}^{26}$. Les pratiques cultuelles, pour Hooker, ont une capacité propre à être lieu de l'action divine rédemptrice, constituante de la vraie Église. Il ne faudrait donc pas, en les réformant trop radicalement, priver le peuple d'un élément important dans la transmission et l'édification de sa foi, quelles que soient par ailleurs les corruptions dont ces pratiques ont pu être entachées dans le passé. Du fait de leur enracinement traditionnel, elles jouissent, chez le commun, d'une autorité (supérieure peut-être à celle d'une doctrine dont il ne saisit pas nécessairement toute la substance) qui fait d'elles des passeurs de la foi catholique et apostolique. À tort ou à raison, Hooker voit le puritanisme de ses adversaires comme une religion de l'abstraction qui semble ignorer que l'Église est une réalité concrète, faite de chair et de sang.

20 Le sacramentalisme très fort de Hooker, évident au livre $\mathrm{V}$ des Lois, va de pair avec son insistance sur l'Église visible. Dans la section christologique préliminaire à son exposition des sacrements, il déclare : 'Our being in Christ by eternal foreknowledge saveth us not without our actual and real adoption in to the fellowship of his saints in this present world [...] We are therefore adopted sons of God to eternal life by participation of the only-begotten son of $\mathrm{God}^{27}$. Or, cette participation au Fils unique de Dieu, pour Hooker, se fait par les sacrements $^{28}$. En restant parfaitement protestant, quelles que soient par ailleurs les critiques qu'il nourrit à l'encontre du prédestinarisme inspiré de Théodore de Bèze, Hooker rappelle quelque chose que n'aurait vraisemblablement pas contredit Calvin : le décret éternel de prédestination ne doit pas mener à être tellement obnubilé par l'appartenance à l'Église invisible qu'on en oublie que cette appartenance, dans le concret, passe par une participation pleine et entière à la vie sacramentelle de l'Église visible et que, en un sens, l'Église invisible, quelle que soit son origine dans le décret éternel de Dieu, n'a pas d'existence sans l'Église visible et ses cérémonies les plus concrètes. Or, celles-ci ont une histoire, histoire de corruptions sans doute, le plus souvent, mais qui n'empêchent pas Dieu d'y opérer le salut.

21 Comme le suggère Rowan Williams, Hooker affirme quelque chose qui détonne peutêtre avec le discours protestant habituel, mais qui est néanmoins ancré dans les principes les plus fondamentaux de la Réforme, à savoir la priorité de l'action de Dieu sur celle des hommes dans l'économie du salut. La manière dont celui-ci s'opère dans le concret de la vie de l'Église est quelque peu brouillonne, et c'est la raison pour laquelle Hooker est en mesure de voir plus de continuité entre l'Église médiévale et l'Église 
élisabéthaine que d'autres théologiens réformés ${ }^{29}$. Il demeure, ce faisant, dans une conversation constructive avec l'orthodoxie protestante: il en élargit peut-être les termes, mais il n'en sort pas véritablement.

\section{Conclusion}

22 C'est une Réforme dans la continuité historique contre une Réforme de la rupture que défend Hooker dans le cadre d'une théologie qui ne doit rien au Concile de Trente. C'est de ce protestantisme-là que se réclame ce qu'on peut commencer à appeler, à partir de la Restauration, l'anglicanisme. Il représente une tradition religieuse anglaise qui occupe un autre espace que celui de l'opposition continentale entre protestantisme et catholicisme, entre tradition religieuse de la rupture et tradition religieuse de la continuité. Pour Hooker, l'hérésie justifie la rupture, mais ne doit pas détruire le sentiment d'appartenance des réformés à la continuité historique de l'Église visible, sans laquelle il n'y aurait pas d'Église invisible.

Ce protestantisme qui revendique sa catholicité historique, et non pas seulement doctrinale ${ }^{30}$, est responsable d'un autre usage des notions de protestantisme et de catholicisme que celui dont on est familier de ce côté-ci de la Manche. Sur le continent, le discours protestant de la rupture, en refusant autant qu'il lui était possible, l'héritage de l'Église médiévale - identifié tout entier à l'hérésie de la Rome tridentine - a permis, la propagande romaine aidant, de présenter l'Église du pape comme seule héritière d'une histoire continue du catholicisme depuis les apôtres. Évoquant les défenseurs de la cause romaine, Hooker écrit: 'They ask us where our Church did lurk, in what cave of the earth it slept for so many hundreds of years together before the birth of Martin Luther?' ${ }^{31} \mathrm{La}$ possibilité même de poser cette question, génération après génération depuis la fin du $\mathrm{XVI}^{\mathrm{e}}$ siècle, a indubitablement participé de la confessionnalisation du concept de catholicisme: celui-ci est devenu propriété de l'Église de Rome contre un protestantisme réformé continental qui avait oublié ses liens avec l'Église médiévale. La tradition hookerienne, très forte au sein de l'anglicanisme, refusant d'opposer en tout l'Église catholique médiévale à l'Église d'Angleterre réformée, a permis à celle-ci de se comprendre comme à la fois protestante et catholique. Non pas parce que son protestantisme serait moins protestant que les autres, mais parce que son discours et sa pratique ont su garder vivante l'idée que le protestantisme est catholicisme dans la continuité de l'histoire de l'Église d'Occident.

\section{NOTES}

1. Nous désignerons cet ouvrage sous le titre abrégé de Laws (ou de Lois, en français). Toutes les citations de Hooker renvoient à W. Speed HILL (ed.), The Folger Library Edition of the Works of Richard Hooker, 7 vols., Cambridge, Mass. : Harvard University Press, 1977-1998. Nous référerons à cette édition de l'œuvre de Hooker par le titre abrégé de Works. Nous avons néanmoins modernisé l'orthographe, dans les citations, pour faciliter la lecture. 
2. Voir notamment Peter LAKE, Anglicans and Puritans?: Presbyterianism and English Conformist Thought from Whitgift to Hooker, London: Unwin Hyman, 1988; Nigel VOAK, Richard Hooker and Reformed Theology: A Study of Reason, Will, and Grace, Oxford: Oxford University Press, 2003; Torrance KIRBY (ed.), A Companion to Richard Hooker, Leiden: Brill, 2008.

3. Rémy BETHMONT, L'Anglicanisme: un modèle pour le christianisme à venir ? Genève : Labor et Fides, 2010, pp. 74-76, 133.

4. Ibid., pp. 26-28.

5. En particulier, son utilisation fréquente non seulement des Pères de l'Église, mais aussi des théologiens médiévaux, notamment saint Thomas d'Aquin. Voir A. S. McGRADE, 'Classical, Patristic and Medieval Sources', in KIRBY, op. cit., pp. 51-87.

6. J'adapte ici une formule de Bernard Cottret, prononcée lors d'un échange sur l'anglicanisme en mai 2012

7. Voir notamment William FULKE, An Answer of a True Christian, reproduit dans Two Treatises written against the Papistes, London, 1577; Oliver CARTER, An Answere unto certaine Popish Quesstions and Demaundes, London, 1579.

8. Richard BAUCKHAM, 'Hooker, Travers and the Church of Rome in the 1580s', Journal of Ecclesiastical History, vol. 29, n 1, January 1978, pp. 44-47.

9. Regroupés et publiés en 1612 sous le titre de A Learned Discourse of Justification, Works, and how the Foundation of Faith is Overthrown.

10. 'We will not judge those, which have died in ignorance for want of knowledge, but refer all to God's secret judgment, who knoweth whom he hath chosen' (CARTER, op. cit., p. 41).

11. 'How then was it possible for so many of our fathers to be saved, sinth they were so far from departing out of Babylon, that they took her for their mother, and in her bosom yielded up the ghost?' (A Learned Discourse of Justification, § 10 [Works, vol. 5, p. 119]).

12. BAUCKHAM, op. cit., pp. 45-48.

13. A Learned Discourse of Justification, $\S 19$ (Works, vol. 5, pp. 126-127).

14. A Learned Discourse of Justification, $\S 17$ (Works, vol. 5, p. 125).

15. A Learned Discourse of Justification, $\S 32$ (Works, vol. 5, pp. 155-156).

16. Deborah K. SHUGER, 'Faith and Assurance', in KIRBY, op.cit., p. 246.

17. Jean CALVIN, Institution de la religion chrestienne, 2 vols., Paris : Ch. Meyrueis, 1859 (livre IV, chap. 2, §12), vol. 2, p. 325.

18. Laws, IV. 8.1 (Works, vol. 1, p. 298).

19. Laws, IV. 5.1 (Works, vol. 1, p. 288).

20. Laws, IV. 9.1 (Works, vol. 1, p. 302). Une étude comparative de cette vision hookerienne et de la Réforme luthérienne serait sans doute bienvenue. Si la distinction avec le monde réformé et puritain est assez nette, elle l'est sans doute beaucoup moins avec le monde luthérien. En 1629, John Donne insiste sur ce point dans un de ses sermons : 'That in all such things [i.e. ceremonies], we always inform ourselves, of the right use of those things in their first institution, of their abuse with which they have been depraved in the Roman Church, and of the good use which is made of them in ours. That because pictures have been adored, we do not abhor a picture; Nor sit at the Sacrament, because idolatry hath been committed in kneeling. That Church, which they call Lutheran, hath retained more of these ceremonies than ours hath done; And ours more than that which they call Calvinist; But both Lutheran, and ours, [are] without danger, because, in both places, we are diligent to preach to the people the right use of these indifferent things' (George POTTER \& Evelyn SIMPSON (eds.), The Sermons of John Donne, 10 vols., Berkeley: University of California Press, 1953-1962, vol. 8 [1956], p. 331).

21. Laws IV. 13.1 (Works, vol. 1, pp. 327-328).

22. S'opposant à l'argument puritain qui veut qu'on guérisse la corruption romaine par son contraire, imposant le bannissement de tout ce qui peut rappeler le papisme, Hooker répond : 'He that will take away extreme heat by setting the body in extremity of cold, shall undoubtedly remove the disease, but together with it the diseased too' (Laws IV. 8. 1 [Works, vol. 1, p. 298]). 
23. Laws IV. 14.1 (Works, vol. 1, pp. 336-337).

24. Hooker se place ici franchement du côté de l'ordre établi et apporte des arguments supplémentaires à la reine, qui considère les demandes puritaines comme une forme de résistance à son autorité.

25. On rappelle que, pour Hooker, l'Église, communauté religieuse, est coextensive à la communauté politique : 'There is not any man of the Church of England, but the same man is also a member of the Commonwealth; nor any man a member of the Commonwealth, which is not also of the Church of England [ ...] properties and actions of one kind do cause the name of a Commonwealth, qualities and functions of another sort, the name of a Church to be given unto a multitude; yet one and the self same multitude may in such sort be both' (Laws, VIII. 1. 2 [Works, vol. 3, p. 319]).

26. Rowan WILLIAMS, 'Hooker the Theologian', Journal of Anglican Studies, vol. 1, nº 1, 2003, p. 109.

27. Laws V. 56.7 (Works, vol. 2, pp. 238-239).

28. Voir en particulier Laws, V. 67. 5-6 (Works, vol. 2, pp. 334-335).

29. WILLIAMS, art. cit., pp. 104-106.

30. Hooker emploie souvent le terme de "catholique", qu'il oppose en général à celui de " hérétique ». Son « catholicisme » est donc d'abord doctrinal, comme chez les autres théologiens réformés. Mais, contrairement à eux, parce qu'il reconnaît une continuité catholique depuis les Pères jusqu'à lui (c'est-à-dire que l'Église n'a jamais été rongée par l'hérésie au point d'en perdre toute catholicité), on peut dire que sa conception de la catholicité est aussi historique.

31. Laws, III. 1. 10 (Works, vol. 1, p. 201).

\section{RÉSUMÉS}

En définissant de manière particulièrement étroite les termes de l'hérésie papiste, quelle que soit par ailleurs sa gravité, Richard Hooker (1554-1600) est amené à voir l'Eglise médiévale d'une manière plus positive que ne la considère la tradition argumentative calviniste. L'hérésie de l'Eglise du pape ne l'a pas totalement empêché d'être, pendant la période médiévale, un passeur de la foi catholique et apostolique. Sans renier les intuitions fondamentales de l'orthodoxie protestante, Hooker inaugure ainsi un protestantisme anglais de la continuité, posant les bases d'une compréhension anglicane de la Réforme comme étant dans la continuité historique du catholicisme occidental.

By closely circumscribing what papist heresy consisted in, however grievous it might be, Richard Hooker (1554-1600) was able to see the medieval Church in a more positive light than was customary in Calvinist discourse. Papist heresy did not constitute a definitive obstacle for the catholic and apostolic faith to be handed down from the apostolic age to the Reformation era. Without overthrowing the fundamentals of Protestant orthodoxy, Hooker started a brand of English Protestantism emphasising continuity with the past, at the basis of the Anglican understanding of the Reformation as being in the historic continuity of Western Catholicism.

\section{AUTEUR \\ RÉMY BETHMONT}

Université de Picardie Jules-Verne 